\title{
Autophagy is involved in neurofibromatosis type I gene-modulated osteogenic differentiation in human bone mesenchymal stem cells
}

\author{
YIQIANG LI, MINGWEI ZHU, XUEMEI LIN, JINGCHUN LI, ZHE YUAN, YANHAN LIU and HONGWEN XU \\ Department of Pediatric Orthopedics, Guangzhou Women and Children's Medical Center, \\ Guangzhou Medical University, Guangzhou, Guangdong 510623, P.R. China
}

Received October 25, 2020; Accepted July 14, 2021

DOI: $10.3892 /$ etm.2021.10697

\begin{abstract}
Neurofibromatosis type I (NF1) is an autosomal dominant genetic disease that is caused by mutations in the $N F 1$ gene. Various studies have previously demonstrated that the mTOR complex 1 signaling pathway is essential for the NF1-modulated osteogenic differentiation of bone mesenchymal stem cells (BMSCs). Additionally, the mTOR signaling pathway plays a notable role in autophagy. The present study hypothesized that NF1 could modulate the osteogenic differentiation of BMSCs by regulating the autophagic activities of BMSCs. In the present study, human BMSCs were cultured in an osteogenic induction medium. The expression of the NFl gene was either knocked down or overexpressed by transfection with a specific small interfering RNA (siRNA) targeting NF1 or the pcDNA3.0 NF1-overexpression plasmid, respectively. Autophagic activities of BMSCs (Beclin-1, P62, LC3B I, and LC3B II) were determined using western blotting, electron microscopy, acridine orange (AO) staining and autophagic flux/lysosomal detection by fluorescence microscopy. In addition, the autophagy activator rapamycin (RAPA) and inhibitor 3-methyladenine (3-MA) were used to investigate the effects of autophagy on NF1-modulated osteogenic differentiation in BMSCs. Inhibiting NF1 with siRNA significantly decreased the expression levels of autophagy markers Beclin-1 and LC3B-II, in addition to osteogenic differentiation markers osterix, runt-related
\end{abstract}

Correspondence to: $\mathrm{Dr}$ Hongwen $\mathrm{Xu}$ or $\mathrm{Dr}$ Yiqiang $\mathrm{Li}$, Department of Pediatric Orthopedics, Guangzhou Women and Children's Medical Center, Guangzhou Medical University, 9 Jinsui Road, Guangzhou, Guangdong 510623, P.R. China

E-mail: xuhongwen@gwcmc.org

E-mail: liyiq@gwcmc.org

Abbreviations: AO, acridine orange; 3-MA, 3-methyladenine; ALP, alkaline phosphatase; BMSCs, bone mesenchymal stem cells; mTORC1, mammalian target of rapamycin complex 1; NF1, neurofibromatosis type I; RAPA, rapamycin

Key words: neurofibromatosis type I, bone marrow stem cells, osteogenic differentiation, autophagy, PI3K/AKT/mTOR pathway transcription factor 2 and alkaline phosphatase. By contrast, overexpressing NF1 with pcDNA3.0 significantly increased their levels. Transmission electron microscopy, AO staining and autophagic flux/lysosomal detection assays revealed that the extent of autophagosome formation was significantly decreased in the NF1-siRNA group but significantly increased in the NF1-pcDNA3.0 group when compared with the NC-siRNA and pcDNA3.0 groups, respectively. In addition, the activity of the PI3K/AKT/mTOR pathway [phosphorylated (p)-PI3K, p-AKT, p-mTOR and p-p70S6 kinase] was significantly upregulated in the NF1-siRNA group compared with the NC-siRNA group, and significantly inhibited in the NF1-pcDNA3.0 group, compared with the pcDNA3.0 group. The knockdown effects of $N F 1$-siRNA on the autophagy and osteogenic differentiation of BMSCs were reversed by the autophagy activator RAPA, while the overexpression effects of NF1-pcDNA3.0 on the autophagy and osteogenic differentiation of BMSCs were reversed by the autophagy inhibitor 3-MA. In conclusion, results from the present study suggest at the involvement of autophagy in the NF1-modulated osteogenic differentiation of BMSCs. Furthermore, NF1 may partially regulate the autophagic activity of BMSCs through the PI3K/AKT/mTOR signaling pathway.

\section{Introduction}

Neurofibromatosis type I (NF1) is an autosomal dominant genetic disorder caused by mutations in the NF1 gene (1-3). The reported incidence of NF1 varies from $1 / 2,500$ to $1 / 3,500$ individuals worldwide (1-3). The clinical manifestations of NF1 are varied and include cafe-au-lait spots, hamartomas of the iris and skeletal abnormalities $(2,4)$. It has been reported that $50 \%$ patients with NF1 have associated skeletal abnormalities, including long bone dysplasia, sphenoid wing dysplasia, scoliosis and congenital pseudarthrosis of the tibia $(5,6)$.

Although mutations in the neurofibromin (NF1) gene are considered to be the primary cause of the occurrence of NF1 $(1,7)$, the mechanism underlying the formation of skeletal abnormalities associated with NF1 is still not fully understood. The NFI gene encodes a Ras GTPase that consists of 2,818 amino acids (1). Mutations in NF1 lead to the functional deficiency of neurofibromin and hyperactivation 
of p21-Ras (1,7). In addition, NF1 has been documented to regulate bone mesenchymal stem cell, neuronal, and glial cell proliferation, differentiation and survival (1).

Previous studies have demonstrated the existence of significantly impaired osteogenic differentiation in human bone mesenchymal stem cells (BMSCs) in patients with NF1-associated skeletal abnormalities $(8,9)$. Furthermore, abnormal osteoblast differentiation and proliferation have been reported to occur due to the loss of NFI function (10-12). A previous study showed that the $N F 1$ gene can modulate the proliferation and osteogenic differentiation of BMSCs (13). In addition, it has been demonstrated that inhibiting the expression of $N F 1$ can activate mTOR complex 1 (mTORC1) signaling and subsequently inhibit the osteogenic differentiation of BMSCs (13).

Autophagy is an evolutionarily conserved adaptive response that takes part in numerous physiological and pathological processes (14). Previous studies have reported that autophagy serves an important role in the osteogenic differentiation of BMSCs $(15,16)$. The PI3K/AKT/mTOR pathway is an important signaling pathway that is involved in the regulation of signal transduction and biological processes, such as cell proliferation, differentiation, apoptosis, metabolism and angiogenesis (17). The PI3K/AKT/mTOR pathway is also considered to be a classical signaling pathway for autophagy activation (18), such that mTORC1 is the main gatekeeper to autophagy that connects environmental cues to metabolic processes in order to preserve cellular homoeostasis (19). In a recent study, Tan et al (20) revealed that overexpression of NFI gene enhanced the osteogenic differentiation of BMSCs by promoting autophagy and that $\mathrm{mTORC} 1$ signaling was involved in this process. However, this previous study only established $N F 1$-overexpression BMSC models, which is different from the clinical situation in patients with $N F 1$, where the function of the NF1 gene is typically insufficient (20). Therefore, a cell model with the inhibited expression of $N F I$ would simulate the pathological conditions of NF1 more closely compared with one modeling the overexpression of NF1.

The present study established cell models of BMSCs that with reduced $N F 1$ expression or overexpressed $N F 1$, similar to the protocol followed in a previous study (13). To investigate the effect of autophagy on the osteogenic differentiation of BMSCs, a classical autophagy inhibitor 3-methyladenine (3-MA) and a specific mTOR inhibitor rapamycin (RAPA), were used. The aims of the present study were as follows: i) To evaluate the effect of $\mathrm{NFl}$ on the autophagy of BMSCs; ii) to investigate the effect of autophagy on NFl-modulated osteogenic differentiation of BMSCs; and iii) to verify the effect of the PI3K/AKT/mTOR signaling pathway on NFI-mediated regulation of BMSC autophagy.

\section{Materials and methods}

Culture of human BMSCs. BMSCs were purchased from the American Type Culture Collection (cat. no. CRL-3421). They were cultured in Human Bone Marrow Mesenchymal Stem Cell Basal Medium (Cyagen Biosciences, Inc.) supplemented with $10 \%$ qualified FBS (Takara Bio, Inc.), $10 \%$ glutamine and $10 \%$ penicillin-streptomycin at $37^{\circ} \mathrm{C}$ in a humidified atmosphere with $5 \% \mathrm{CO}_{2}$ for 14 days (13). The standard procedure to induce osteogenic differentiation of BMSCs is by culturing cells in Human Mesenchymal Stem Cell Osteogenic Differentiation Basal Medium (Cyagen Biosciences, Inc.) containing 10\% FBS (Takara Bio, Inc.), $0.2 \%$ ascorbate acid (Shanghai Aladdin Biochemical Technology Co., Ltd.), 0.01\% dexamethasone (Shanghai Aladdin Biochemical Technology Co., Ltd.), $1 \%$ glutamine, 100 units of penicillin/streptomycin and $1 \% \beta$-glycerophosphate sodium (13).

Cell transfection and treatment. BMSC models with inhibited or overexpressed $N F 1$ were established using a method similar to that used by a previous study (13). Briefly, a small interfering RNA (siRNA) targeting $N F 1$ and a negative control (NC) siRNA (non-targeting sequence) were purchased from Shanghai GenePharma Co., Ltd. The siRNA targeting sequences were as follows: $N F 1$-siRNA, 5'-ACATACCAAAGTCAGTAC T-3'; and NC-siRNA, 5'-AACAAGATGAAGAGCACCA-3'. Human BMSCs were grown in 6/12-well culture plates until $\sim 80 \%$ confluence, then transfected with $100 \mathrm{~nm}$ siRNA-NFI or NC-siRNA for $48 \mathrm{~h}$ at $37^{\circ} \mathrm{C}$ using Lipofectamine ${ }^{\circledR} 2000$ (Invitrogen; Thermo Fisher Scientific, Inc.). Human NFI cDNA was amplified by PCR and inserted into pcDNA3.0 (NF1-pcDNA3.0; Invitrogen; Thermo Fisher Scientific, Inc.). Then NF1-pcDNA3.0 $(1 / 2 \mu \mathrm{g})$ or pcDNA3.0 $(1 / 2 \mu \mathrm{g})$ was transfected into BMSCs using Lipofectamine ${ }^{\circledR} 2000$ in accordance with the manufacturer's protocol. Twenty-four hours later, the transfected cells were divided into the following four groups: NC-siRNA, NF1-siRNA, pcDNA3.0 and NF1-pcDNA3.0. Additionally, a control group was established containing untransfected BMSCs. The transfected BMSCs were treated with 50 nM RAPA (cat. no. AY 22989; MedChemExpress) or $5 \mathrm{mM}$ 3-MA (cat. no. S2767-1; Selleck Chemicals) for $24 \mathrm{~h}$ at $37^{\circ} \mathrm{C}$ for the detection of autophagy and associated pathways. The concentrations used in the present study were based on those used in previous studies $(21,22)$. In addition, osteogenic differentiation experiments were conducted on day 14 following transfection.

$R N A$ extraction and reverse transcription-quantitative PCR $(R T-q P C R)$. Following transfection and differentiation on day 14 , total RNA was isolated from cultured cells using TRIzol ${ }^{\circledR}$ reagent (Invitrogen; Thermo Fisher Scientific, Inc.) according to the manufacturer's instructions. Gene expression levels were measured in a real-time PCR detection system (Bio-Rad Laboratories, Inc.) by SYBR ${ }^{\circledR}$ Green (Bio-Rad Laboratories, Inc.) detection. Briefly, the extracted RNA was reverse transcribed in the presence of a poly (A) polymerase with an oligo-dT adaptor, using the PrimeScript ${ }^{\mathrm{TM}} \mathrm{RT}$ Reagent kit with gDNA Eraser (Perfect Real Time; cat. no. RR047A; Takara Bio, Inc.), according to the manufacturer's protocol. The thermocycling conditions were as follows: $42^{\circ} \mathrm{C}$ for $2 \mathrm{~min}$; followed by $37^{\circ} \mathrm{C}$ for $15 \mathrm{~min}$ and $85^{\circ} \mathrm{C}$ for $5 \mathrm{sec}$. The expression of $N F 1$, runt-related transcription factor 2 (Runx2), alkaline phosphatase (ALP) and Osterix were quantified by qPCR using TB Green ${ }^{\circledast}$ Fast qPCR Mix (cat. no. RR430; Takara Bio, Inc.). Thermocycling conditions were as follows: Initial denaturation at $95^{\circ} \mathrm{C}$ for $15 \mathrm{~min}$; followed by 40 cycles of $95^{\circ} \mathrm{C}$ for $15 \mathrm{sec}$, annealing $50-60^{\circ} \mathrm{C}$ for $30 \mathrm{sec}, 72^{\circ} \mathrm{C}$ for $30 \mathrm{sec}$; followed by final extension at $72^{\circ} \mathrm{C}$ for $7 \mathrm{~min}$. GAPDH was used as the internal control. The PCR primers are listed 
Table I. Primers used in the present study.

\begin{tabular}{lcl}
\hline ID & Orientation & \multicolumn{1}{c}{ Sequence (direction, 5'-3') } \\
\hline NF1 & F & GTATTGAATTGAAGCACCTTTGTTTGG \\
NF1 & R & CTGCCCAAGGCTCCCCCAG \\
ALP & F & CCAACTCTTTTGTGCCAGAGA \\
ALP & R & GGCTACATTGGTGTTGAGCTTTT \\
Runx2 & F & GACTGTGGTTACCGTCATGGC \\
Runx2 & R & ACTTGGTTTTTCATAACAGCGGA \\
Osterix & F & ACCTACCC ATCTGACTTTGCTC \\
Osterix & R & CTGCCCACTATTTCCCACTG \\
GAPDH & F & AGGTCGGTGTGAACGGATTTG \\
GAPDH & $\mathrm{R}$ & GGGGTCGTTGATGGCAACA
\end{tabular}

NF1, neurofibromatosis type I; ALP, alkaline phosphatase; Runx2, runt-related transcription factor 2; F, forward; R, reverse.

in Table I. The Cq value obtained for the gene of interest was normalized to that of the housekeeping gene GAPDH to obtain the $\Delta \mathrm{Cq}$ value. The $\Delta \Delta \mathrm{Cq}$ value was then obtained by subtracting the $\Delta \mathrm{Cq}$ value for each gene of interest from the $\Delta \mathrm{Cq}$ value for the control sample. The results were calculated using the equation $R Q=2^{-\Delta \Delta C q}$, where $R Q$ is the relative quantity and is expressed as the fold-change relative to the corresponding gene expression level in the control sample (23).

Western blotting. Transfected and treated BMSC proteins were extracted using lysis buffer containing $50 \mathrm{mM}$ Tris (pH 7.6), $150 \mathrm{mM} \mathrm{NaCl}, 1 \%$ Triton X-100, $1 \%$ deoxycholate, $0.1 \%$ SDS, $1 \mathrm{mM}$ PMSF and $0.2 \%$ aprotinin (Beyotime Institute of Biotechnology). A BCA ${ }^{\mathrm{TM}}$ Protein Assay kit (Pierce; Thermo Fisher Scientific, Inc.) was used for quantification of protein samples. Equal amounts of protein samples (30 $\mu \mathrm{g}$ per lane) were separated on $15 \%$ SDS-PAGE gels and transferred onto PVDF membranes. After blocking in 5\% BSA serum (Roche Diagnostics $\mathrm{GmbH}$ ) for $1 \mathrm{~h}$ at $37^{\circ} \mathrm{C}$, the blocked membranes were incubated with the corresponding primary antibodies overnight at $4^{\circ} \mathrm{C}$. The membranes were then washed in Tris-buffered saline (Tris $20 \mathrm{mM}, \mathrm{NaCl} 137 \mathrm{mM}$, pH 7.6) containing $0.1 \%$ Tween-20 (TBST; cat. no. P2287; Sigma-Aldrich; Merck KgaA) three times and incubated for $1 \mathrm{~h}$ at room temperature with appropriate secondary antibodies conjugated to horseradish peroxidase (HRP). The membranes were incubated with ECL reagent (Immun-Star HRP Substrate kit; cat. no. 1705040; Bio-Rad Laboratories, Inc.). The signals were then visualized and analyzed using Image Lab $^{\text {TM }}$ software 5.2 (Bio-Rad Laboratories, Inc.). For the present study, primary antibodies against NF1 (1:1,000; cat. no. ab128054; Abcam), Beclin-1 (1:1,000; cat. no. ab210498; Abcam), p62 (1:1,000; cat. no. ab109012; Abcam), LC3BI/II (1:1,000; cat. no. ab192890; Abcam), GAPDH (1:1,000; cat. no. ab8245; Abcam), ALP (1:1,000; cat. no. ab229126; Abcam), Runx2 (1:1,000; cat. no. ab236639; Abcam) and Osterix (1:1,000; cat. no. ab209484; Abcam) were used. Antibodies against phosphorylated (p)-mTOR (1:1,000; cat. no. 5536; Cell Signaling Technology), total (t)-mTOR (1:1,000; cat. no. 2983; Cell Signaling Technology),
p-p70S6 kinase (p70S6K; 1:1,000; cat. no. 9204; Cell Signaling Technology), t-p70S6K (1:1,000; cat. no. 2708; Cell Signaling Technology), AKT (1:1,000; cat. no. 9272; Cell Signaling Technology), p-AKT (1:1,000; cat. no. 4060; Cell Signaling Technology), PI3K (1:1,000; cat. no. 4249; Cell Signaling Technology) and p-PI3K (1:1,000; cat. no. 17366; Cell Signaling Technology) were also used. HRP-conjugated anti-mouse $\operatorname{IgG}(1: 5,000$; cat. no. ab6728; Abcam) and HRP-conjugated anti-rabbit IgG (1:5,000; cat. no. ab6721; Abcam) were used as secondary antibodies.

Acridine orange $(A O)$ staining. After conditioning, BMSCs were harvested and suspended in PBS at $1 \times 10^{6}$ cells $/ \mathrm{ml}$. Next, $95 \mu \mathrm{l}$ of this cell suspension was considered and $5 \mu \mathrm{l} \mathrm{AO}$ staining solution (Sigma-Aldrich; Merck KGaA) was added. The reaction was allowed to proceed for $10 \mathrm{~min}$ in the dark at room temperature. Subsequently, $5 \mathrm{ml}$ PBS was added and the suspension was then centrifuged on a conventional centrifuge at $150 \mathrm{x} \mathrm{g}$ at room temperature for $5 \mathrm{~min}$. The supernatant was then discarded and washed twice with PBS. The cells suspended in PBS were pipetted onto slides and sealed with cover glass. Autophagy was visualized under a fluorescence microscope (magnification, x40; Leica DMIRB; Leica Microsystems $\mathrm{GmbH}$ ).

Autophagic flux/lysosomal detection. To track and observe the formation of autophagosomes and autophagic flux, $1 \times 10^{6}$ cells $/ \mathrm{ml}$ were grown to $\sim 80 \%$ confluence, infected with Ad-GFP-LC3B (cat. no. C3006; Beyotime Institute of Biotechnology) at $7 \log _{10}$ PFU/ml for $24 \mathrm{~h}$ and cultured in a 6-well plate on cover glass to monitor autophagy flux. Furthermore, the slides were washed with PBS and 3\% paraformaldehyde was added into each well. The plate was then placed in the dark for $20 \mathrm{~min}$ at room temperature. The slides were washed three times with PBS before $2 \mathrm{ml}$ PBS was then added to each well. The plate was incubated with shaking at room temperature for $10 \mathrm{~min}$. The coverslips were mounted with an anti-fade mounting solution (cat. no. P0126; Beyotime Institute of Biotechnology) and dried for $1 \mathrm{~min}$ at room temperature. Subsequently, the slides were observed with a fluorescence microscope 
(magnification, x40; Leica DMIRB; Leica Microsystems $\mathrm{GmbH}$ ). During this procedure, GFP-LC3 was combined with autophagosomes, and detected using fluorescence microscopy. Under the fluorescence microscope, the GFP-LC3 combined autophagosomes were indicated by granular green fluorescence.

ALP staining. BMSCs were inoculated into a 12 -well culture plate at a density of $1 \times 10^{4}$ cells per well at $37^{\circ} \mathrm{C}$ in $5 \% \mathrm{CO}_{2}$. After $24 \mathrm{~h}$, the medium was replaced with the osteogenic induction medium (cat. no. CTCC-Y001, PH Biomedicine) and cultured at $37^{\circ} \mathrm{C}$ in $5 \% \mathrm{CO}_{2}$. The medium was changed every $2-3$ days and removed after 14 days. The cells were washed twice with PBS and fixed with $4 \%$ paraformaldehyde for $30 \mathrm{~min}$ at room temperature. The paraformaldehyde was then removed and the cells were washed three times with $\mathrm{ddH}_{2} \mathrm{O}$ and an ALP staining solution (Shanghai Gefan Biotechnology Co., Ltd.) was added for $30 \mathrm{~min}$ at room temperature. The ALP staining solution was removed, the cells were washed three times with $\mathrm{ddH}_{2} \mathrm{O}$ and visualized under a microscope (magnification, x40; Leica DMIRB; Leica Microsystems GmbH); in addition, images were captured.

Alizarin red staining. BMSCs were cultured with osteogenic induction medium for 14 days before examination of Alizarin red staining. The culture medium was discarded and the cells $\left(1 \times 10^{6}\right)$ were fixed with $4 \%$ paraformaldehyde for $15-20 \mathrm{~min}$ at room temperature, following which they were washed three times with PBS. Alizarin red staining solution (ScienCell Research Laboratories, Inc.) was prepared in advance and was added to the culture plate and placed in the incubator for $15 \mathrm{~min}$ at room temperature. The staining solution was then discarded, the plate was washed three times with PBS solution and placed under a differential interference contrast microscope (magnification, x40; Leica DMIRB; Leica Microsystems $\mathrm{GmbH}$ ) to capture images.

Transmission electron microscopy. BMSCs were cultured in 6-well plates and transfected with NF1-pcDNA3.0 for $48 \mathrm{~h}$, and then collected and fixed with a mixture of $2.5 \%$ glutaraldehyde and $1 \%$ acetic acid for $2 \mathrm{~h}$ at room temperature. Samples were then processed following a standard protocol (24). Briefly, samples were dehydrated using ethanol, stained for $2 \mathrm{~h}$ using uranyl acetate and alkaline lead citrate at room temperature, embedded using Epon resin at $37^{\circ} \mathrm{C}$ overnight, and cut into $500 \mathrm{~nm}$-thick sections using an automatic microwave sample processor (Leica EM AMW; Leica Microsystems GmbH) at room temperature. Observation and imaging were then performed by using a JEM-1400 transmission electron microscope (JEOL, Ltd.).

Statistical analysis. All experiments were repeated $\geq$ three times. The results are expressed as the mean \pm standard deviation. Data were processed using the SPSS 10.0 statistical software (SPSS, Inc.). For between-group comparisons, data were analyzed using an unpaired Student's t-test. One-way analysis of variance followed by a post hoc test of LSD was used to analyze the data among the three groups. $\mathrm{P}<0.05$ was considered to indicate a statistically significant difference.

\section{Results}

NF1 regulates the autophagic activity of BMSCs. To detect the effect of $N F 1$ on the autophagic activity of BMSCs, cell models of BMSCs with NF1 knockdown using NF1-siRNA or NF1 overexpression (NF1-pcDNA3.0) o were established. RT-qPCR and western blotting results showed that the mRNA (Fig. 1A) and protein (Fig. 1B and C) expression levels of NF1 were significantly decreased in the NF1-siRNA group compared with those in NC siRNA, but significantly increased in the NF1-pcDNA3.0 group compared with those in the pcDNA3.0 group. Western blotting results indicated that in the NF1-siRNA group, the expression of Beclin-1 was significantly decreased, whereas that of LC3B-I and p62, a marker of autophagosome degradation (25), were significantly increased compared with that in the NC siRNA group (Fig. 1B and C). In addition, in the NF1-pcDNA3.0 group, the expression of autophagy markers Beclin-1, was significantly increased, whereas that of LC3B-I and p62 was significantly decreased compared with that in the pcDNA3.0 group (Fig. 1B and C).

Furthermore, autophagic activity was detected using transmission electron microscopy. The results revealed that overexpression of NF1(NF1-pcDNA3.0) increased the formation of autophagosomes, whilst inhibiting the expression of NF1(NF1-siRNA) significantly reduced the formation of autophagosomes when compared with groups pcDNA3.0 and NC-siRNA, respectively (Fig. 2). Furthermore, AO staining and autophagic flux/lysosomal detection were performed (Fig. 3A). AO staining demonstrated that the formation of autophagolysosomes (indicated by yellow-red or orange fluorescence in Fig. 3A) was increased in the NF1-pcDNA3.0 group but decreased in the $N F 1$-siRNA group when compared with groups NC-siRNA and pcDNA3.0. The autophagic flux/lysosomal detection assay also revealed similar results (Fig. 3B). During the formation of autophagosomes, GFP-LC3 protein transferred to the membrane of autophagosomes, and the autophagosomes were indicated by green puncta using fluorescence microscopy. The results of the present study indicated that the autophagic flux/lysosomal was significantly increased in the NF1-pcDNA3.0 group but decreased in the NF1-siRNA group when compared with the pcDNA3.0 and NC-siRNA groups, respectively.

Collectively, these results suggest that overexpression of $N F 1$ promoted autophagic activity of BMSCs, whilst knockdown of NF1 expression decreased the level of autophagy in BMSCs.

NF1 modulates osteogenic differentiation by influencing the autophagic activity of BMSCs. The results of RT-qPCR and western blotting indicated that the expression levels of osteogenic differentiation markers Osterix, Runx2 and ALP were markedly decreased in the NF1-siRNA group, whereas they were increased in the NF1-pcDNA3.0 group compared with those in the NC siRNA group (Fig. 4A-D). To investigate the effects of autophagy on osteogenic differentiation of BMSCs, an activator (RAPA) and inhibitor (3-MA) of autophagy were added to the NF1-siRNA and NF1-pcDNA3.0 groups, respectively. As a result, the levels of the osteogenic differentiation markers Osterix, Runx2 and ALP increased markedly in the RAPA-treated group compared with that in 

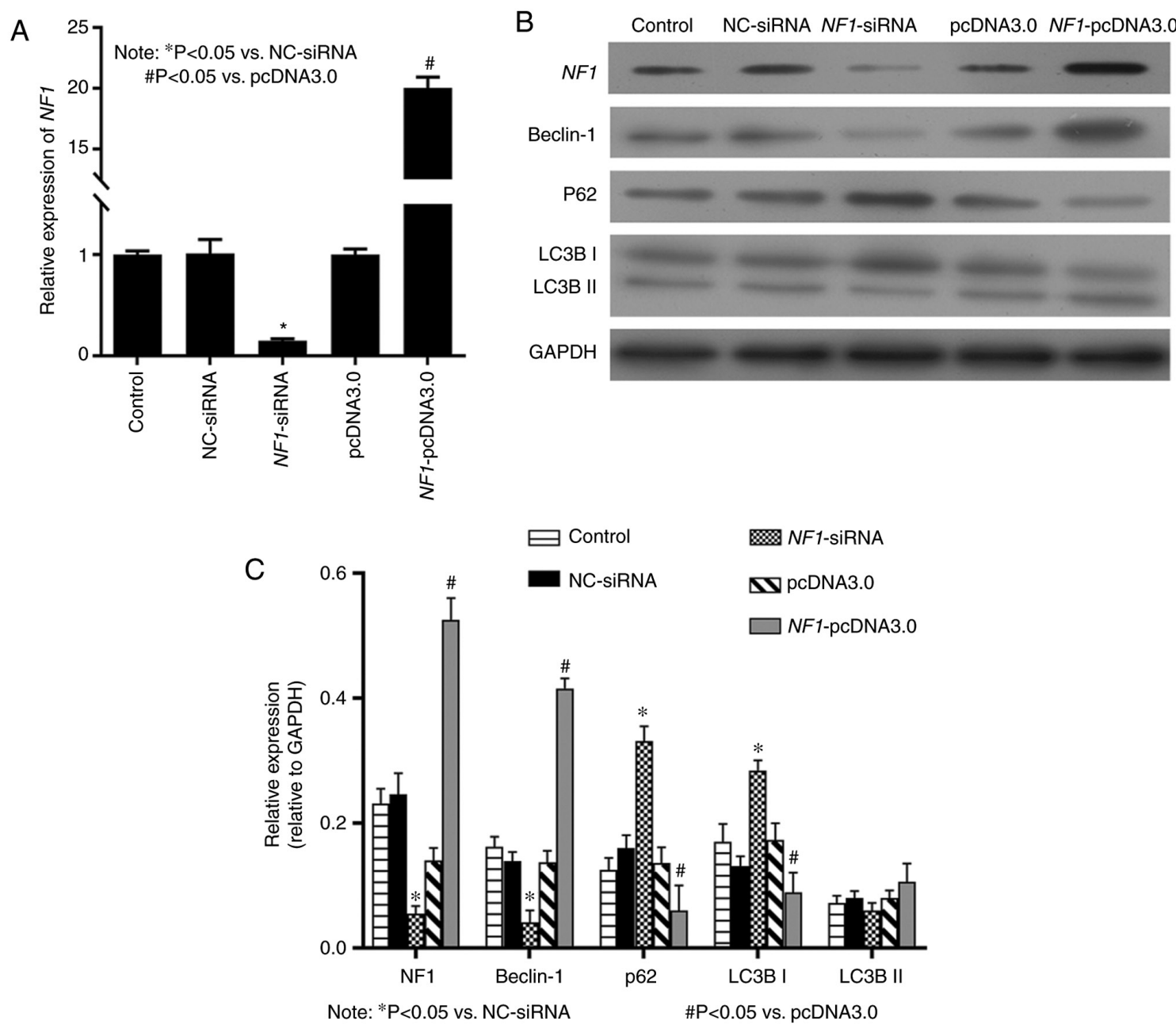

Figure 1. NF1 regulates the autophagic activity of bone mesenchymal stem cells. (A) Reverse transcription-quantitative PCR demonstrated significant downregulation of $N F 1$-mRNA expression in the $N F 1$-siRNA group and upregulation of $N F 1$-mRNA expression in the $N F 1$-pcDNA3.0 group. (B) Western blotting and subsequent (C) quantification indicated that the protein levels of $N F 1$, Beclin-1 were significantly decreased in the $N F 1$-siRNA group and increased in the NF1-pcDNA3.0 group, whereas the changes in the expression of p62 and LC3B-I revealed the opposite pattern. ${ }^{*} \mathrm{P}<0.05$ vs. NC-siRNA; ${ }^{*} \mathrm{P}<0.05$ vs. NF1-pcDNA3.0. NF1, neurofibromin-1; si-, small interfering; NC, negative control.
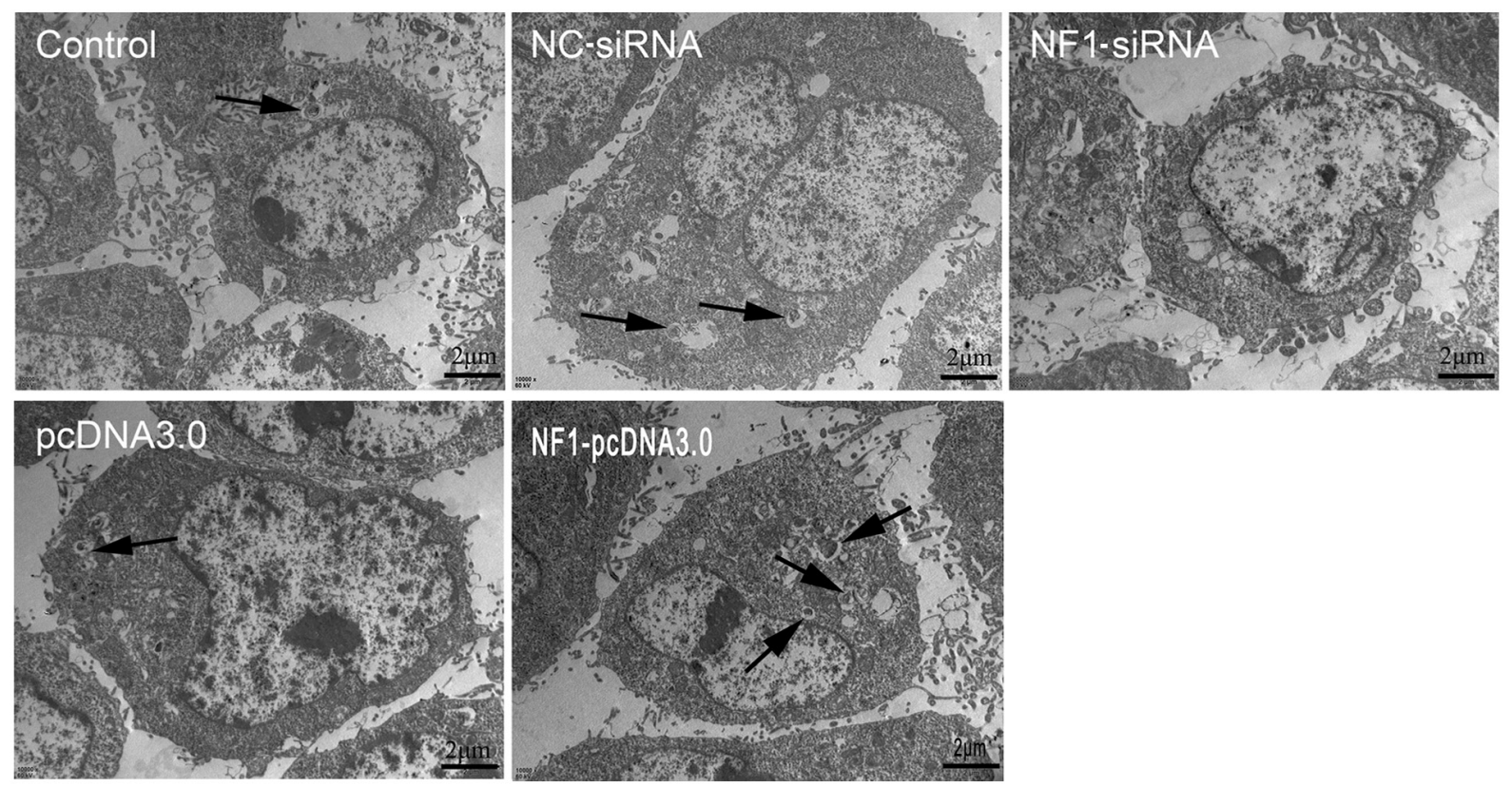

Figure 2. Transmission electron microscopy results. It demonstrated that the formation of autophagosomes was decreased in the NF1-siRNA group and increased in the NF1-pcDNA3.0 group (indicated by the arrows). Scale bars, $2 \mu \mathrm{m}$. NC, negative control; si-, small interfering; $N F 1$, neurofibromin-1. 
A
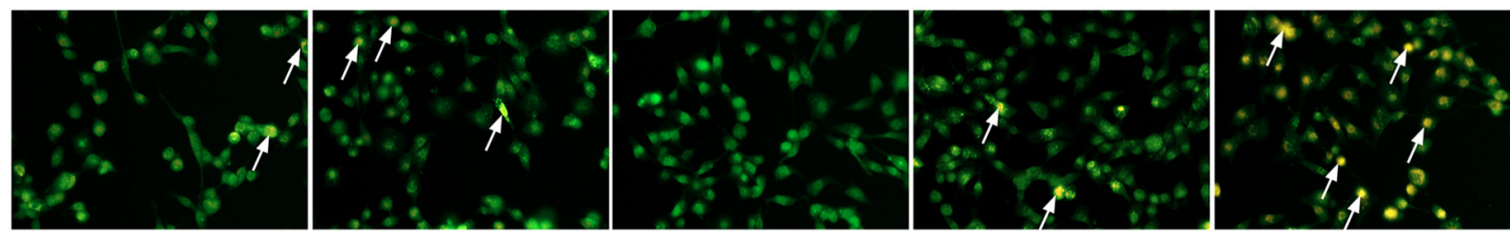

Control

NC-siRNA

NF1-siRNA

pcDNA3.0

NF1-pcDNA3.0

B
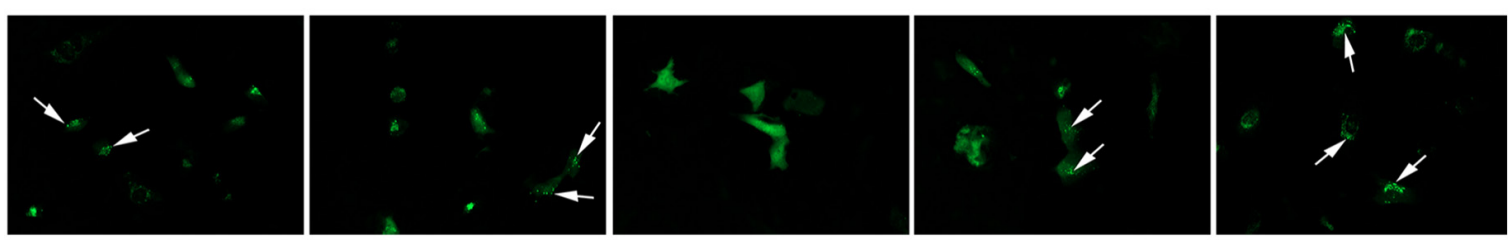

Figure 3. AO staining and autophagic flux detection from the Ad-GFP-LC3 visualized under a fluorescence microscope. (A) AO staining, autophagolysosomes were stained orange (indicated by white arrows), (B) GFP-LC3 combined autophagosomes were indicated by granular green fluorescence (indicated by the white arrows). Magnification, x200. AO, acridine orange; NF1, neurofibromin-1; si-, small interfering; NC, negative control.
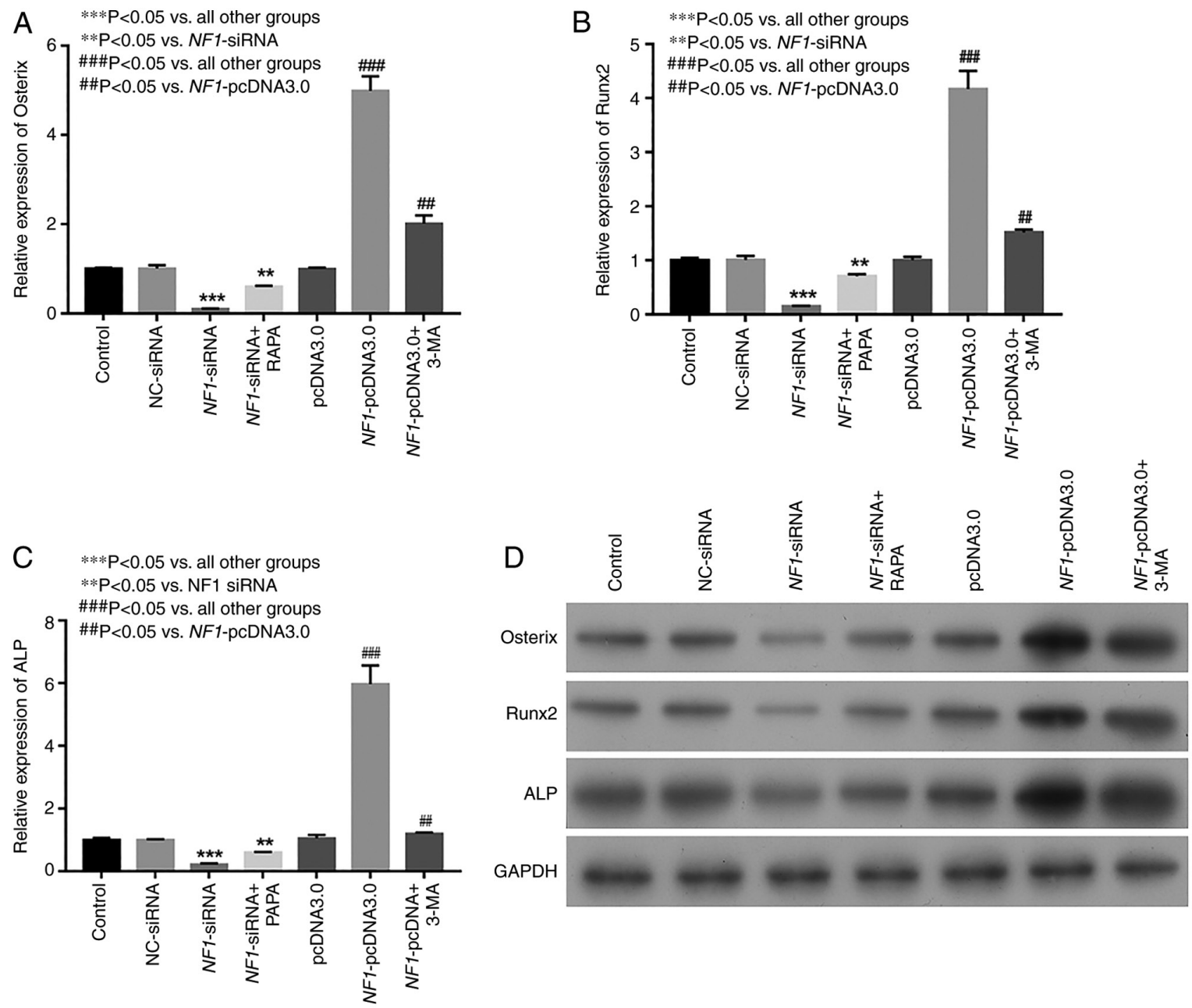

Figure 4. Autophagy serves a notable role in NF1-modulated osteogenic differentiation of bone mesenchymal stem cells. Expression levels of osteogenic markers (A) Osterix, (B) Runx2 and (C) ALP measured by reverse transcription-quantitative PCR. (D) Western blotting indicated that the expression levels of these markers were markedly decreased in the NF1-siRNA group and increased in the NF1-pcDNA3.0 group. (A-D) Treatment with RAPA and 3-MA markedly but partially reversed the effects of $N F 1$-siRNA and NF1-pcDNA3.0 transfection, respectively. NF1; neurofibromin-1; si-, small interfering; NC, negative control; Runx2, runt-related transcription factor 2; ALP, alkaline phosphatase; RAPA, rapamycin; 3-MA, 3-methyladenine; ${ }^{* * *} \mathrm{P}<0.05$ vs. all other groups;

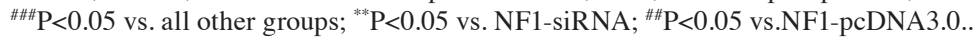

the NF1-siRNA group alone. By contrast, the expression levels decreased markedly in the 3-MA-treated group compared with those in $N F 1$-pcDNA3.0 group alone (Fig. 4A-D).
ALP staining indicated that ALP activity was decreased by knocking down $N F 1$ expression (NF1-siRNA) and increased by NF1 overexpression (NF1-pcDNA3.0) when compared 

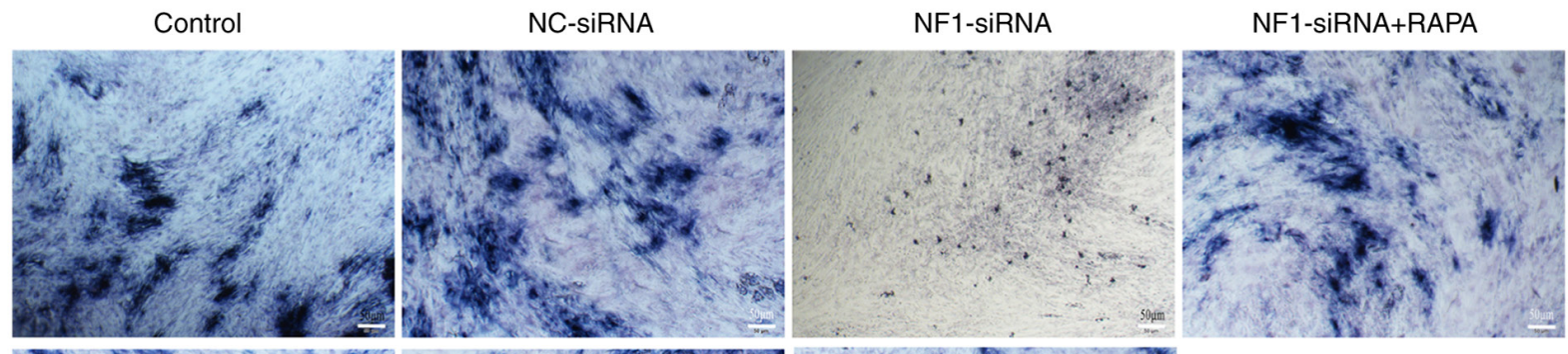

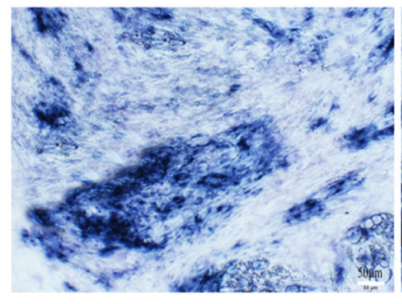

pcDNA3.0

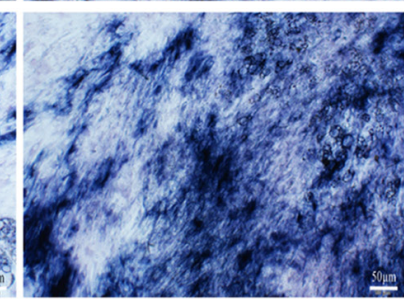

NF1-pcDNA3.0

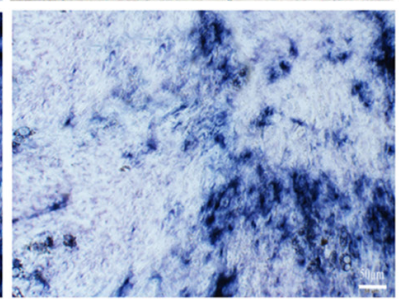

NF1-pcDNA3.0+3-MA

Figure 5. Results of ALP staining. Activity of ALP was markedly decreased in the NF1-siRNA group and increased in the NF1-pcDNA3.0 group when compared with NC-siRNA and pcDNA3.0 groups, respectively. However, treatment with RAPA and 3-MA reversed the effects of NF1-siRNA and NF1-pcDNA3.0 transfection, respectively. Scale bars, $50 \mu \mathrm{m}$. NF1, neurofibromin-1; si-, small interfering; NC, negative control; ALP, alkaline phosphatase; RAPA, rapamycin; 3-MA, 3-methyladenine.

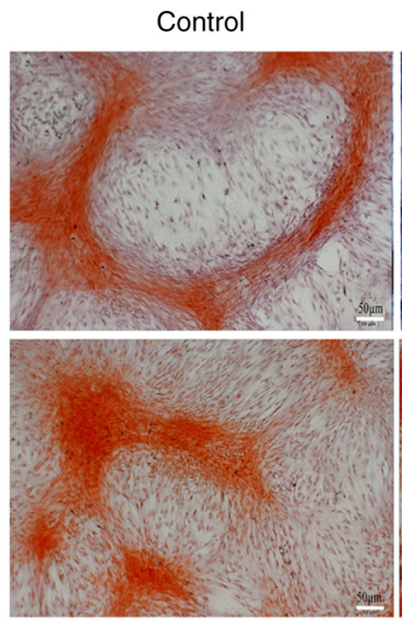

pcDNA3.0

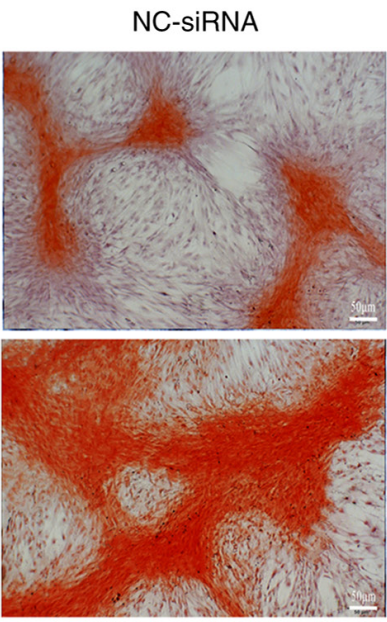

NF1-pcDNA3.0
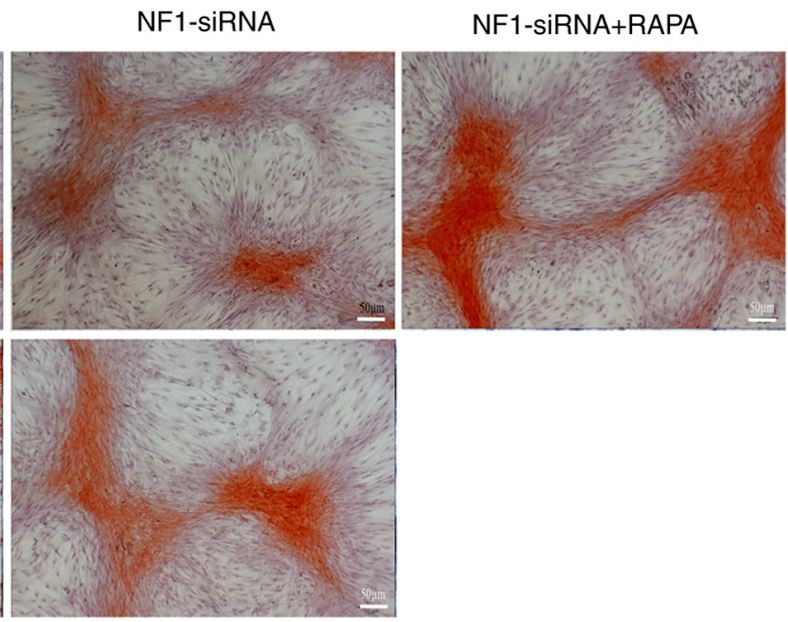

NF1-pcDNA3.0+3-MA

Figure 6. Alizarin red staining. Calcium deposition was decreased in the $N F 1$-siRNA group and increased in the $N F 1$-pcDNA3.0 group when compared with NC-siRNA and pcDNA3.0 groups, respectively. Treatment with RAPA and 3-MA reversed the effects of $N F 1$-siRNA and NF1-pcDNA3.0 transfection, respectively. Scale bars, $50 \mu \mathrm{m}$. NF1, neurofibromin-1; si-, small interfering; NC, negative control; RAPA, rapamycin; 3-MA, 3-methyladenine.

with NC-siRNA and pcDNA3.0 groups, respectively. In addition, RAPA and 3-MA treatment reversed the changes in ALP activity in the NF1-siRNA and NF1-pcDNA3.0 groups, respectively (Fig. 5). Alizarin red staining, representing calcium deposition, also revealed similar results (Fig. 6). After application of RAPA and 3-MA, the decreased and increased calcium deposition in the NF1-siRNA and NF1-pcDNA3.0 groups, respectively, were restored to the levels in the control groups (Fig. 6). These results suggest that NF1 could modulate the osteogenic differentiation of BMSCs by regulating the autophagic activity of BMSCs.

NF1 partially regulates the autophagic activity of BMSCs via the PI3K/AKT/mTOR pathway. The present study verified whether NF1 could regulate autophagy in BMSCs through the PI3K/AKT/mTOR pathway. Western blotting results demonstrated that the $\mathrm{PI} 3 \mathrm{~K} / \mathrm{AKT} / \mathrm{mTOR}$ pathway was significantly activated in the NF1-siRNA group, as indicated by increased levels of p-PI3K, p-AKT, p-mTOR and p-p70S6K compared with those in the NC-siRNA group (Fig. 7A-C). By contrast, the PI3K/AKT/mTOR pathway was significantly inhibited in the $N F 1$-pcDNA3.0 group, as indicated by decreased levels of p-PI3K, p-AKT, p-mTOR and p-p70S6K compared with those in the pcDNA3.0 group (Fig. 7A-C). RAPA was used to activate whereas 3-MA was used to inhibit the autophagy of BMSCs in the NF1-siRNA and NF1-pcDNA3.0 groups, respectively. The results of western blot analysis indicated that the autophagic activity was significantly increased in group NF1-siRNA+RAPA and decreased in group NF1-pcDNA3.0+3-MA, compared with the NF1-siRNA and group NF1-pcDNA3.0, respectively (Fig. 7D-F). Transmission electron microscopy, AO staining 
A

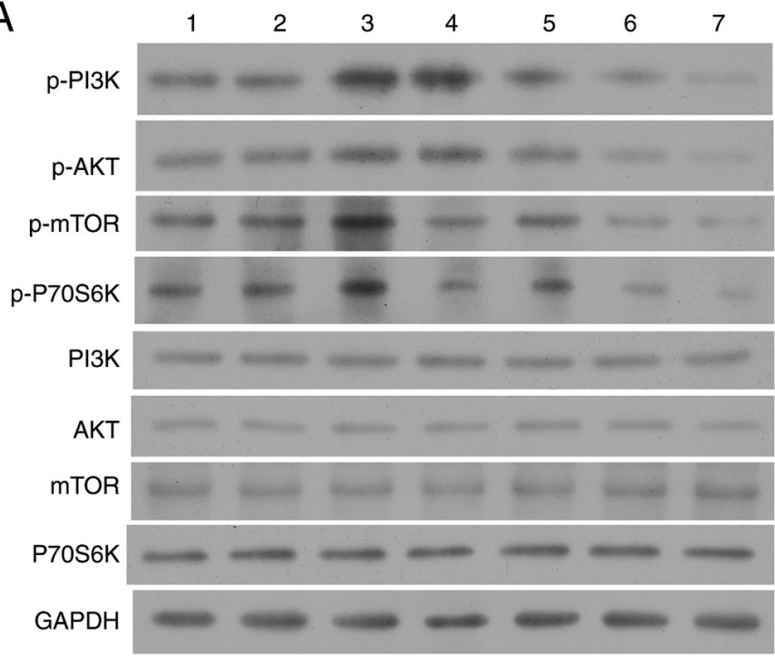

1: Control

2: NC-siRNA

3: NF1-siRNA

4: NF1-siRNA+RAPA

5: pcDNA3.0

6: NF1-pcDNA3.0

7: NF1-pcDNA3.0+MA

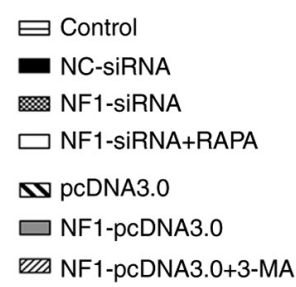

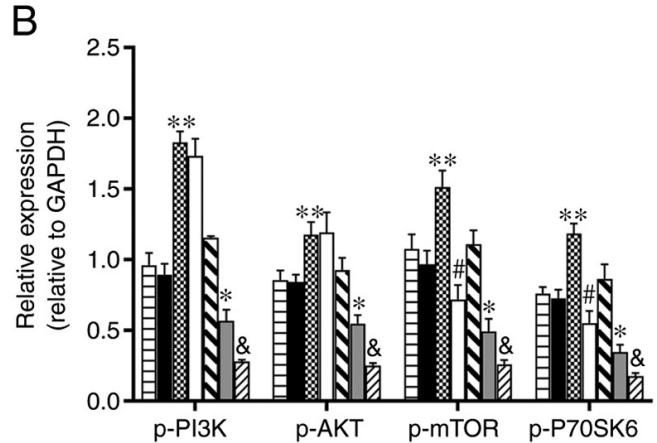

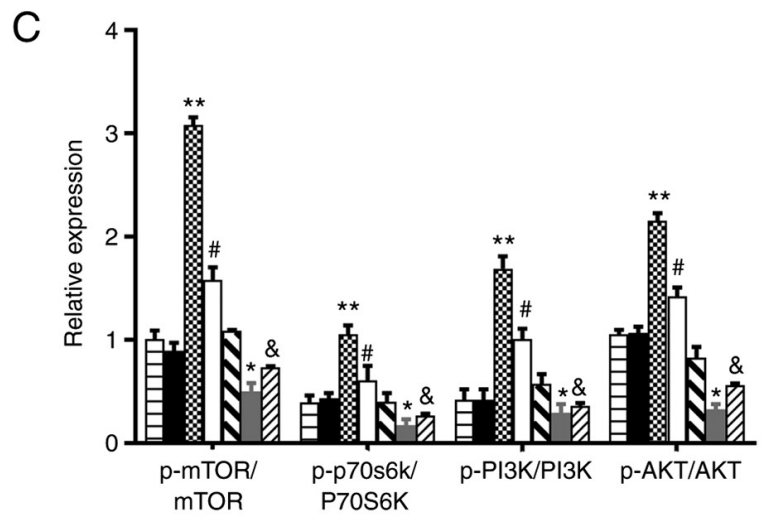

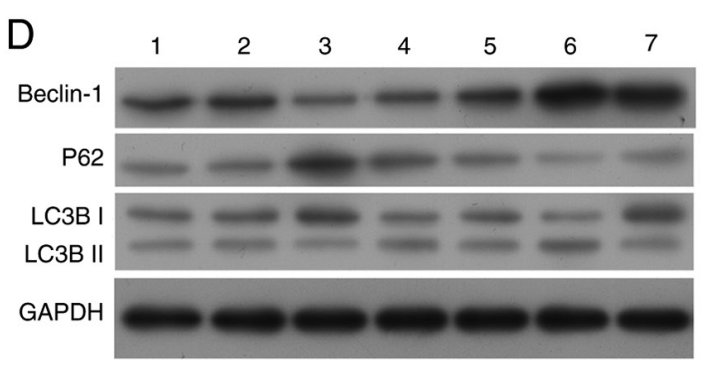

E

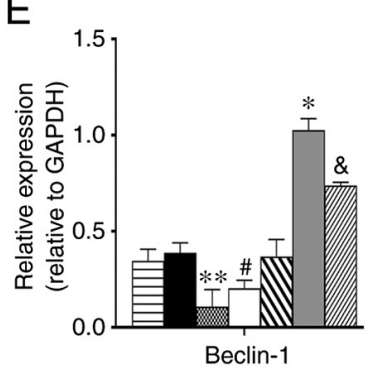

$\mathrm{F}$

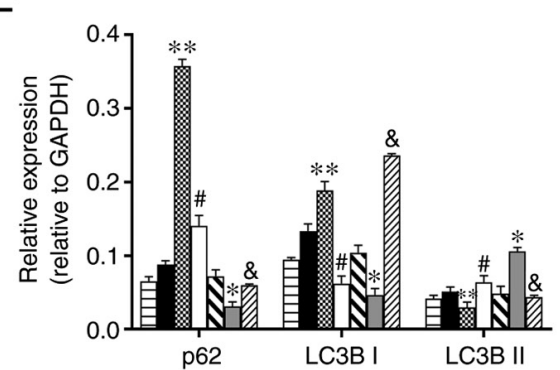

Note: ${ }^{* *} \mathrm{P}<0.05$ vs. NC-siRNA; \#P<0.05 vs. NF1-siRNA; ${ }^{*} \mathrm{P}<0.05$ vs. pcDNA3.0; \&P<0.05 vs. NF1-pcDNA3.0

Figure 7. NF1 regulates autophagy in bone mesenchymal stem cells by inhibiting the PI3K/AKT/mTOR pathway. (A-C) Western blotting demonstrated that $N F 1$-siRNA significantly decreased the expression of Beclin-1 and LC3B-II and increased the expression of P62 and LC3B-I, while RAPA reversed the effects of NF1-siRNA. NF1-pcDNA3.0 significantly increased the expression of Beclin-1 and LC3B-II while decreased the expression of P62 and LC3B-I, while 3-MA reversed the effects of NF1-pcDNA3.0. (D-F) Western blotting indicated that the levels of proteins in the PI3K/AKT/mTOR pathway (p-PI3K, p-AKT, p-mTOR and p-P70S6K) were upregulated in the NF1-siRNA group and downregulated in the NF1-pcDNA3.0 group when compared with groups NC-siRNA and pcDNA3.0, respectively. RAPA downregulated PI3K/AKT/mTOR pathway in BMSCs with NF1-siRNA, while 3-MA upregulated PI3K/AKT/mTOR pathway in BMSCs with NF1-pcDNA3.0. Quantified levels of (B) Beclin-1, (C) p62, LC3B-I, LC3B-II, (E, F) p-PI3K, p-AKT, p-mTOR and p-P70S6K. ${ }^{*} \mathrm{P}<0.05$ vs. pcDNA3.0; ${ }^{* *} \mathrm{P}<0.05$ vs NC-siRNA; ${ }^{\mathrm{P}}<0.05$ vs. NF1-siRNA; ${ }^{\circ} \mathrm{P}<0.05$ vs. NF1-pcDNA3.0. NF1, neurofibromatosis type 1; si-, small interfering; $\mathrm{NC}$, negative control; RAPA, rapamycin; 3-MA, 3-methyladenine; p-, phosphorylated; P70S6K, p70S6 kinase.

and autophagic flux/lysosomal detection indicated that the formation of autophagosomes were significantly increased in the NF1-siRNA + RAPA group compared with the $N F 1$-siRNA, whilst it was significantly decreased in the $N F 1$-pcDNA3.0 + 3-MA group compared with that in the NF1-pcDNA3.0 group (Figs. 8 and 9). The levels of p-mTOR, p-p70S6K, p-PI3K, and p-AKT were significantly decreased in the NF1-siRNA + RAPA group compared with those in the NF1-siRNA group (Fig. 7A-C). Additionally, the levels of p-PI3K, p-AKT, p-mTOR and p-p70S6K were all significantly decreased in the NF1-pcDNA3.0 + 3-MA group compared with those in the NF1-pcDNA3.0 group (Fig. 7A-C). These results indicated that NF1 could partially regulate the autophagic activity of BMSCs via the PI3K/AKT/mTOR signaling pathway.

\section{Discussion}

Data from the present study indicate that $N F 1$ regulated the autophagy of BMSCs. Overexpression of NF1 promoted the autophagic activity of BMSCs, whilst autophagic activity was inhibited by the downregulation of NF1. Several studies have reported the effect of autophagy on NF1-deficient malignant peripheral nerve sheath tumors (MPNSTs) $(26,27)$. In particular, Yang et al (26) found that NF1-deficient MPNST samples exhibit high mobility group protein A2 

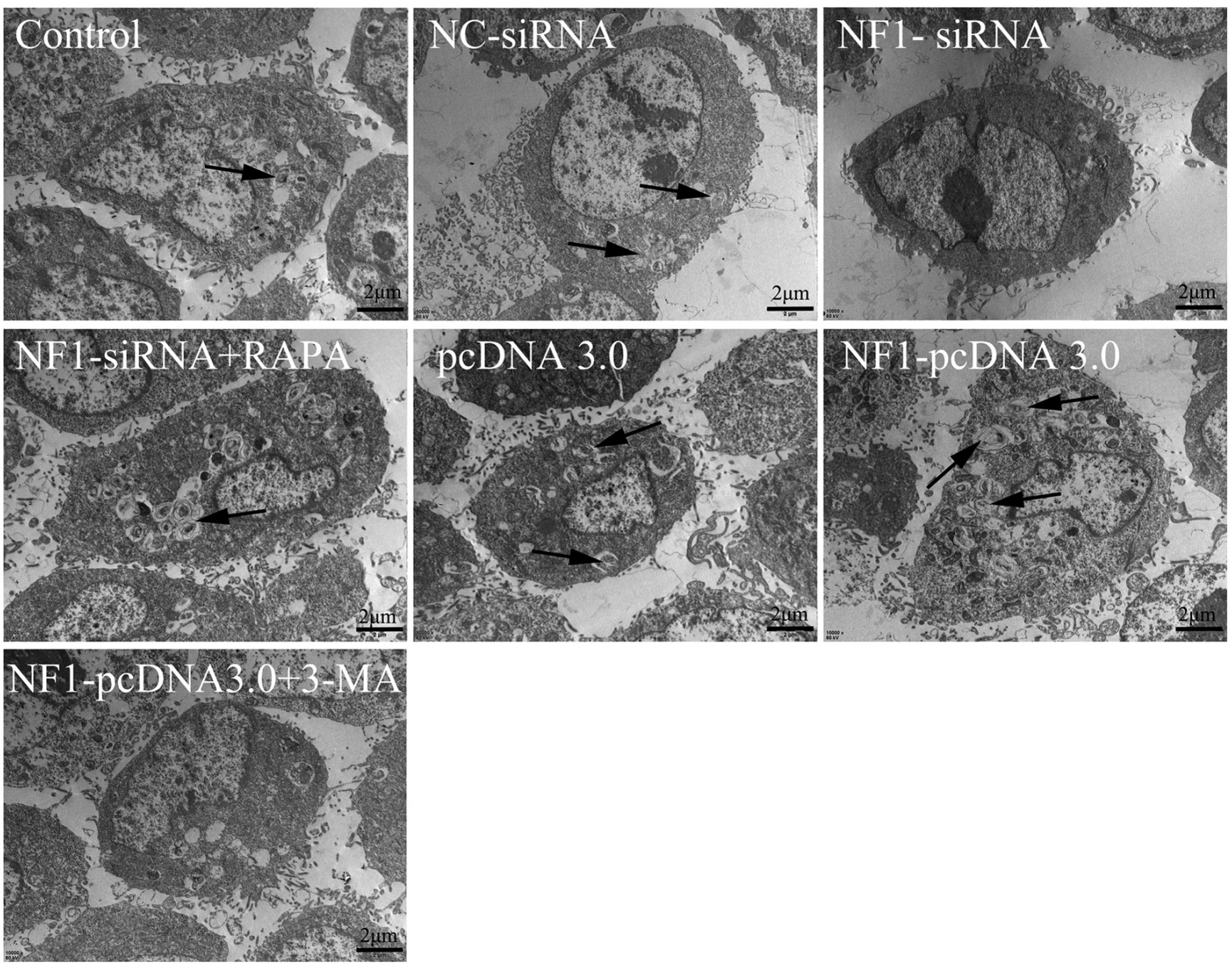

Figure 8. Results of transmission electron microscopy. Treatment with RAPA and 3-MA reversed the effects of $N F 1$-siRNA and NF1-pcDNA3.0 transfection, respectively, on the autophagy activity of BMSCs (autophagosome was indicated by the arrows). Scale bars, $2 \mu \mathrm{m} . N F 1$, neurofibromin-1; si-, small interfering; NC, negative control; RAPA, rapamycin; 3-MA, 3-methyladenine.

(HMGA2) expression levels and that HMGA2 knockdown inhibited autophagy, which subsequently promotes MPNST cell death. However, to the best of our knowledge, few studies have reported the effects of NFI on autophagy in BMSCs. Tan et al (20) revealed that autophagic activity and osteogenic differentiation were significantly enhanced in NFI-overexpressing BMSCs, consistent with the results of the present study. However, Tan et al (20) only established NFI-overexpression BMSC models, which are different from the clinical situation in patients with NF1 mutations, where the function of $N F 1$ is insufficient (8). Therefore, a cell model with inhibited expression of $N F I$ can simulate the pathological conditions of NF1 more closely compared with one with overexpression of NF1. The present study established cell models of BMSCs with the inhibition or overexpression of $N F 1$, which is more translational for investigating the effects of NFl on the autophagy of BMSCs $(26,27)$.

The present study also demonstrated that autophagy served a notable role in NFI-modulated osteogenic differentiation of BMSCs. Knockdown of NF1 inhibited the autophagic activity of BMSCs and decreased the osteogenic differentiation of BMSCs whilst overexpression of $\mathrm{NFI}$ resulted in the opposite effects. In addition, an autophagy activator (RAPA) and an autophagy inhibitor (3-MA) reversed the effects of $\mathrm{NFl-knockdown}$ and overexpression, respectively, on the osteogenic differentiation of BMSCs. A number of studies have demonstrated the involvement of NF1 in osteogenic differentiation of BMSCs $(8,10,11,28)$. In particular, a previous study demonstrated that downregulation and upregulation of $N F 1$ respectively inhibited and promoted osteogenic differentiation of BMSCs, respectively (13). Leskelä et al (8) cultured mesenchymal stem cells of patients with NF1 and revealed impaired osteoblast differentiation. Conversely, loss of NFI resulted in increased osteoblast proliferation $(10,11)$. Kolanczyk et al (10) established a mouse model with conditional inactivation of NFI in the limb skeleton and tested the effect of NFI on osteoblast proliferation. They revealed that the osteoblast cell division rate was significantly increased in inactivated $N F 1$ mutant cells when compared with that in controls. Additionally, other studies have demonstrated that autophagy can serve an important role in the osteogenic differentiation process $(15,16,29-32)$. Wan et al (15) investigated the lumbar BMSCs of patients with osteoporosis and determined 

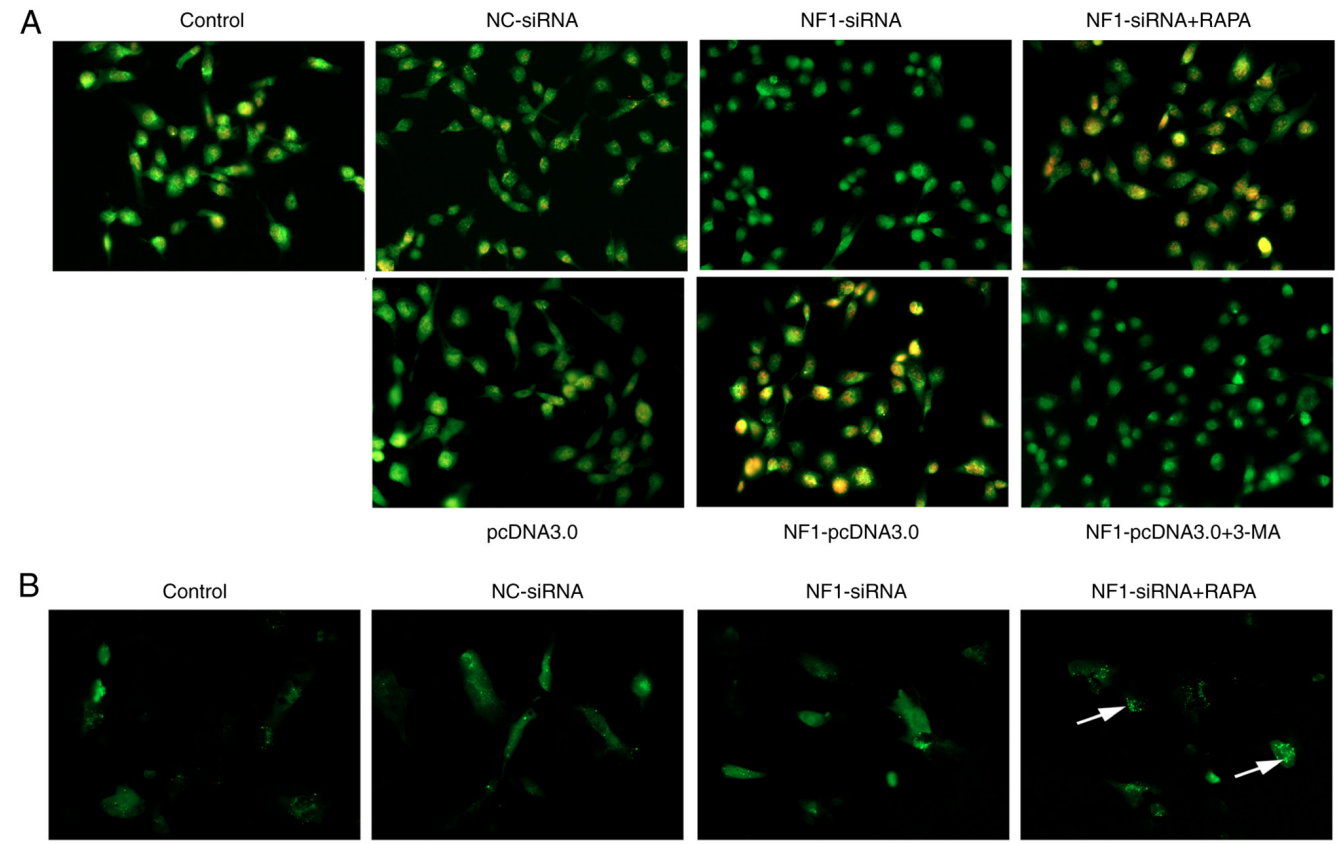

NF1-pcDNA3.0+3-MA
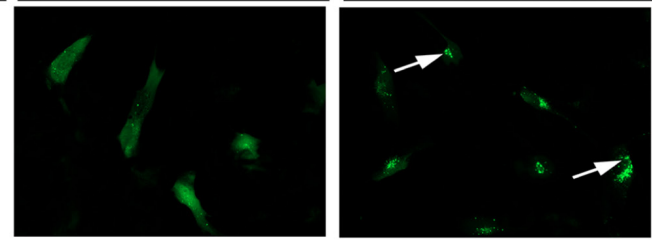

NF1-pcDNA3.0

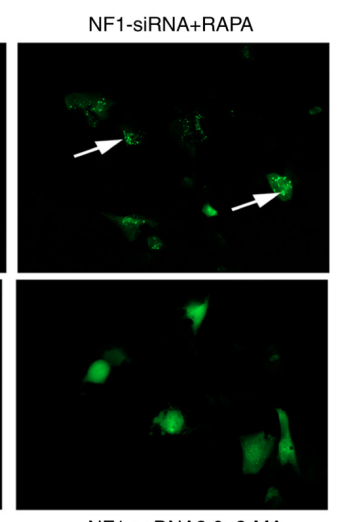

NF1-pcDNA3.0+3-MA

Figure 9. AO staining and autophagic flux detection visualized under a fluorescence microscope. Magnification, x200. AO staining and autophagic flux detection visualized under a fluorescence microscope. In AO staining (A), autophagolysosomes were stained orange (indicated by the white arrows). The formation of autophagosomes were significantly increased in the $N F 1$-siRNA + RAPA group compared with the NF1-siRNA, whilst it was significantly decreased in the NF1-pcDNA3.0 + 3-MA group compared with that in the NF1-pcDNA3.0 group. In autophagic flux detection (B), the GFP-LC3 combined autophagosomes were indicated by granular green fluorescence (indicated by the white arrows). Autophagic flux was significantly increased in the $N F 1$-siRNA + RAPA group compared with the NF1-siRNA group, while it was significantly decreased in the NF1-pcDNA3.0 + 3-MA group compared with that in the NF1-pcDNA3.0 group. Results indicated that treatment with RAPA and 3-MA reversed the effects of $N F 1$-siRNA and $N F 1$-pcDNA3.0 transfection, respectively (indicated by the arrows). AO, acridine orange; NF1, neurofibromin 1; si-, small interfering; NC, negative control; RAPA, rapamycin; 3-MA, 3-methyladenine.

that the autophagy level in these BMSCs was significantly decreased, which was accompanied by inhibited osteogenic differentiation. Furthermore, it was identified that an autophagy activator (RAPA) significantly increased the osteogenic differentiation of BMSCs (15). Ma et al (29) also determined that the autophagy levels in young male mice were higher compared with those in aged male mice. In addition, treatment with an autophagy inhibitor significantly suppressed the osteogenic differentiation of BMSCs (29). Nuschke et al (30) found that autophagy levels in BMSCs were significantly increased during the early stages of osteogenic differentiation, whilst it was significantly decreased after differentiation into mature osteocytes. In addition, Liu et al (31), Gómez-Puerto et al (32) and Zhou et al (16) reported that autophagy served an important role in osteogenic differentiation of BMSCs. In brief, the majority of previous studies aforementioned reported that an increase in autophagic activity promotes osteogenic differentiation of BMSCs. However, the BMSCs used in these previous studies were purchased from patients without NF1 mutation and with a native levels of $N F 1$ expression. The present study used NF1-knockdown and NF1-overexpressing BMSCs and confirmed the involvement of autophagy in NF1-modulated osteogenic differentiation of BMSCs.
The present study also indicated that the autophagic activity of BMSCs was partially regulated by $N F 1$ via the $\mathrm{PI} 3 \mathrm{~K} / \mathrm{AKT} / \mathrm{mTOR}$ signaling pathway. The PI3K/AKT/mTOR pathway was activated in NFl-knockdown BMSCs, whilst it was inhibited in NF1-overexpressing BMSCs. It has been previously reported that the PI3K/AKT/mTOR pathway serves a notable role in regulating autophagy $(18,33,34)$. It is a classical signaling pathway for autophagy activation and the main gateway to autophagy $(18,19)$. In particular, various studies have reported activation of the PI3K/AKT/mTOR signaling pathway in NF1-related MPNSTs $(35,36)$. Previous studies by Tan et al (20) and Li et al (13), combined with the present study, demonstrate that regulation of autophagy facilitates NF1-mediated modulation of osteogenic differentiation of BMSCs via the PI3K/AKT/mTOR signaling pathway.

The mTORC1 signaling pathway has been reported to be an important regulator of autophagy (19). Unc-51-like autophagy activating kinase (ULK) is a key initiator of autophagy $(37,38)$. Furthermore, mTORC1 inhibits the ULK complex by phosphorylating its components, including autophagy-related gene 13 and $\operatorname{ULK} 1 / 2(37,38)$. Additionally, mTORC1 regulates the Vps34 class III PI3K complex, which is needed for autophagosome formation $(37,38)$. In the present 
study, the autophagic activity in the NF1-pcDNA3.0 group was significantly decreased after an autophagy inhibitor (3-MA) was applied. It is known that 3-MA is an inhibitor of PI3K (39) and that it suppresses autophagy by inhibiting the class III PI3K (40). Additionally, 3-MA inhibits AKT by inhibiting the class I PI3K $(40,41)$ and subsequently leads to mTORC1 inactivation (18). As aforementioned, mTORC1 signaling inhibits autophagosome formation $(37,38)$. The inhibition of autophagy and osteogenic differentiation of BMSCs in the NF1-pcDNA3.0 + 3-MA group in the present study suggests that autophagy may partially regulate osteogenic differentiation of BMSCs through other mechanisms that are independent of mTOR signaling. It has been reported that AMP-activated protein kinase and the $\mathrm{Wnt} / / \beta$-catenin signaling pathway may also mediate important roles in regulating autophagy in BMSCs $(42,43)$. Therefore, further study is required to investigate the mechanism by which NF1 modulates osteogenic differentiation of BMSCs through autophagy.

There are still some limitations in the present study. Firstly, our study only established BMSCs models with knockdown or overexpression of $N F 1$ by siRNA or pcDNA3.0, which is different from clinical condition (NF1 mutation). Secondly, the present study is a cell experiment, further animal studies are required to confirm the role of autophagy on NF1-modulated growth of bone.

In conclusion, the present study demonstrated that autophagy played a significant role in NF1-mediated osteogenic differentiation of BMSCs. Downregulation of NF1 inhibited autophagy to decrease osteogenic differentiation of BMSCs, whereas upregulation of NFI activated autophagy to increase osteogenic differentiation. NF1 may partially regulate the autophagic activity of BMSCs via the PI3K/AKT/mTOR signaling pathway. The present study could guide a new direction for elucidating the etiology of NFl-associated skeletal abnormalities and provide a novel theoretical basis for the treatment of NF1.

\section{Acknowledgements}

Not applicable.

\section{Funding}

The present study was supported by funding from Guangzhou Women and Children's Medical Center/Guangzhou Institute of Pediatrics (grant no. IP-2019-001) and The National Nature Science Foundation of China (grant no. 81702116).

\section{Availability of data and materials}

The datasets used and/or analyzed during the current study are available from the corresponding author on reasonable request.

\section{Authors' contributions}

Conceptualization, methodology, supervision and writing (review and editing) were performed by YQL, HWX and XML. Original draft and funding acquisition were performed by YQL. HWX and YQL provided resources. XML, MWZ,
JCL, ZY and YHL performed the experiments. YQL and MWZ analyzed the data. YQL and HWX confirm the authenticity of all the raw data. All authors have read and approved the final manuscript.

\section{Ethics approval and consent to participate}

Not applicable.

\section{Patient consent for publication}

Not applicable.

\section{Competing interests}

The authors declare that they have no competing interests.

\section{References}

1. Anderson JL and Gutmann DH: Neurofibromatosis type 1. Handb Clin Neurol 132: 75-86, 2015.

2. Ferner RE and Gutmann DH: Neurofibromatosis type 1 (NF1): Diagnosis and management. Handb Clin Neurol 115: 939-955, 2013.

3. Gutmann DH, Ferner RE, Listernick RH, Korf BR, Wolters PL and Johnson KJ: Neurofibromatosis type 1. Nat Rev Dis Primers 3: 17004, 2017.

4. Ferner RE, Huson SM, Thomas N, Moss C, Willshaw H, Evans DG Upadhyaya M, Towers R, Gleeson M, Steiger C and Kirby A: Guidelines for the diagnosis and management of individuals with neurofibromatosis 1. J Med Genet 44: 81-88, 2007.

5. Feldman DS, Jordan C and Fonseca L: Orthopaedic manifestations of neurofibromatosis type 1. J Am Acad Orthop Surg 18: 346-357, 2010

6. Vitale MG, Guha A and Skaggs DL: Orthopaedic manifestations of neurofibromatosis in children: An update. Clin Orthop Relat Res 107-118, 2002.

7. Shen MH, Harper PS and Upadhyaya M: Molecular genetics of neurofibromatosis type 1 (NF1). J Med Genet 33: 2-17, 1996.

8. Leskelä HV, Kuorilehto T, Risteli J, Koivunen J, Nissinen M, Peltonen S, Kinnunen P, Messiaen L, Lehenkari P and Peltonen J: Congenital pseudarthrosis of neurofibromatosis type 1: Impaired osteoblast differentiation and function and altered NF1 gene expression. Bone 44: 243-250, 2009.

9. Sharma R, Wu X, Rhodes SD, Chen S, He Y, Yuan J, Li J, Yang X, Li X, Jiang L, et al: Hyperactive Ras/MAPK signaling is critical for tibial nonunion fracture in neurofibromin-deficient mice. Hum Mol Genet 22: 4818-4828, 2013.

10. Kolanczyk M, Kossler N, Kuhnisch J, Lavitas L, Stricker S, Wilkening U, Manjubala I, Fratzl P, Sporle R, Herrmann BG, et al: Multiple roles for neurofibromin in skeletal development and growth. Hum Mol Genet 16: 874-886, 2007.

11. Wu X, Estwick SA, Chen S, Yu M, Ming W, Nebesio TD, Li Y, Yuan J, Kapur R, Ingram D, et al: Neurofibromin plays a critical role in modulating osteoblast differentiation of mesenchymal stem/progenitor cells. Hum Mol Genet 15: 2837-2845, 2006.

12. Wang W, Nyman JS, Ono K, Stevenson DA, Yang X and Elefteriou F: Mice lacking Nf1 in osteochondroprogenitor cells display skeletal dysplasia similar to patients with neurofibromatosis type I. Hum Mol Genet 20: 3910-3924, 2011.

13. Li Y, Li J, Zhou Q, Liu Y, Chen W and Xu H: mTORC1 signaling is essential for neurofibromatosis type I gene modulated osteogenic differentiation of BMSCs. J Cell Biochem 120: 2886-2896, 2019.

14. Reggiori F and Klionsky DJ: Autophagy in the eukaryotic cell. Eukaryot Cell 1: 11-21, 2002.

15. Wan Y,Zhuo N, Li Y, Zhao W and Jiang D: Autophagy promotes osteogenic differentiation of human bone marrow mesenchymal stem cell derived from osteoporotic vertebrae. Biochem Biophys Res Commun 488: 46-52, 2017.

16. Zhou Z, Shi G, Zheng X, Jiang S and Jiang L: Autophagy activation facilitates mechanical stimulation-promoted osteoblast differentiation and ameliorates hindlimb unloading-induced bone loss. Biochem Biophys Res Commun 498: 667-673, 2018. 
17. Lim HJ, Crowe $\mathrm{P}$ and Yang JL: Current clinical regulation of $\mathrm{PI} 3 \mathrm{~K} / \mathrm{PTEN} / \mathrm{Akt} / \mathrm{mTOR}$ signalling in treatment of human cancer. J Cancer Res Clin Oncol 141: 671-689, 2015.

18. Heras-Sandoval D, Perez-Rojas JM, Hernandez-Damian J and Pedraza-Chaverri J: The role of PI3K/AKT/mTOR pathway in the modulation of autophagy and the clearance of protein aggregates in neurodegeneration. Cell Signal 26: 2694-2701, 2014.

19. Rabanal-Ruiz Y, Otten EG and Korolchuk VI: mTORC1 as the main gateway to autophagy. Essays Biochem 61: 565-584, 2017.

20. Tan Q, Wu JY, Liu YX, Liu K, Tang J, Ye WH, Zhu GH, Mei HB and Yang G: The neurofibromatosis type I gene promotes autophagy via mTORC1 signalling pathway to enhance new bone formation after fracture. J Cell Mol Med 24: 11524-11534, 2020

21. Zhao F, Feng G, Zhu J, Su Z, Guo R, Liu J, Zhang H and Zhai Y: 3-Methyladenine-enhanced susceptibility to sorafenib in hepatocellular carcinoma cells by inhibiting autophagy. Anticancer Drugs 32: 386-393, 2021.

22. Song C, Song C and Tong F: Autophagy induction is a survival response against oxidative stress in bone marrow-derived mesenchymal stromal cells. Cytotherapy 16: 1361-1370, 2014.

23. Livak KJ and Schmittgen TD: Analysis of relative gene expression data using real-time quantitative PCR and the 2(-Delta Delta C(T)) method. Methods 25: 402-408, 2001.

24. Klionsky DJ, Abdelmohsen K, Abe A, Abedin MJ, Abeliovich H, Acevedo Arozena A, Adachi H, Adams CM, Adams PD, Adeli $\mathrm{K}$, et al: Guidelines for the use and interpretation of assays for monitoring autophagy (3rd edition). Autophagy 12: 1-222, 2016

25. Shibutani ST, Saitoh T, Nowag H, Münz C and Yoshimori T: Autophagy and autophagy-related proteins in the immune system. Nat Immunol 16: 1014-1024, 2015.

26. Yang K, Guo W, Ren T, Huang Y, Han Y, Zhang H and Zhang J: Knockdown of HMGA2 regulates the level of autophagy via interactions between MSI2 and Beclin1 to inhibit NF1-associated malignant peripheral nerve sheath tumour growth. J Exp Clin Cancer Res 38: 185, 2019.

27. Lopez G, Torres K, Liu J, Hernandez B, Young E, Belousov R, Bolshakov S, Lazar AJ, Slopis JM, McCutcheon IE, et al: Autophagic survival in resistance to histone deacetylase inhibitors: Novel strategies to treat malignant peripheral nerve sheath tumors. Cancer Res 71: 185-196, 2011

28. de la Croix Ndong J, Stevens DM, Vignaux G, Uppuganti S, Perrien DS, Yang X, Nyman JS, Harth E and Elefteriou F: Combined MEK inhibition and BMP2 treatment promotes osteoblast differentiation and bone healing in Nf1Osx-/-mice. J Bone Miner Res 30: 55-63, 2015.

29. Ma Y, Qi M, An Y, Zhang L, Yang R, Doro DH, Liu W and Jin Y: Autophagy controls mesenchymal stem cell properties and senescence during bone aging. Aging Cell 17: e12709, 2018.

30. Nuschke A, Rodrigues M, Stolz DB, Chu CT, Griffith L and Wells A: Human mesenchymal stem cells/multipotent stromal cells consume accumulated autophagosomes early in differentiation. Stem Cell Res Ther 5: 140, 2014.

31. Liu X, Wang Y, Cao Z, Dou C, Bai Y, Liu C, Dong S and Fei J: Staphylococcal lipoteichoic acid promotes osteogenic differentiation of mouse mesenchymal stem cells by increasing autophagic activity. Biochem Biophys Res Commun 485: 421-426, 2017
32. Gomez-Puerto MC, Verhagen LP, Braat AK, Lam EW, Coffer PJ and Lorenowicz MJ: Activation of autophagy by FOXO3 regulates redox homeostasis during osteogenic differentiation. Autophagy 12: 1804-1816, 2016.

33. Chang H, Li X, Cai Q, Li C, Tian L, Chen J, Xing X, Gan Y, Ouyang W and Yang Z: The PI3K/Akt/mTOR pathway is involved in CVB3-induced autophagy of HeLa cells. Int J Mol Med 40: 182-192, 2017.

34. Gao Y, Zhang Y and Fan Y: Eupafolin ameliorates lipopolysaccharide-induced cardiomyocyte autophagy via PI3K/AKT/mTOR signaling pathway. Iran J Basic Med Sci 22: 1340-1346, 2019.

35. Schulte A, Ewald F, Spyra M, Smit DJ, Jiang W, Salamon J, Jucker $M$ and Mautner VF: Combined targeting of AKT and mTOR inhibits proliferation of human NF1-associated malignant peripheral nerve sheath tumour cells in vitro but not in a xenograft mouse model in vivo. Int J Mol Sci 21: 1548, 2020.

36. Li XX, Zhang SJ, Chiu AP, Lo LH, Huang J, Rowlands DK, Wang J and Keng VW: Targeting of AKT/ERK/CTNNB1 by DAW22 as a potential therapeutic compound for malignant peripheral nerve sheath tumor. Cancer Med 7: 4791-4800, 2018.

37. Kim YC and Guan KL: mTOR: A pharmacologic target for autophagy regulation. J Clin Invest 125: 25-32, 2015.

38. Hosokawa N, Hara T, Kaizuka T, Kishi C, Takamura A, Miura Y, Iemura S, Natsume T, Takehana K, Yamada N, et al: Nutrient-dependent mTORC1 association with the ULK1-Atg13-FIP200 complex required for autophagy. Mol Biol Cell 20: 1981-1991, 2009.

39. Blommaart EF, Krause U, Schellens JP, Vreeling-Sindelarova $H$ and Meijer AJ: The phosphatidylinositol 3-kinase inhibitors wortmannin and LY294002 inhibit autophagy in isolated rat hepatocytes. Eur J Biochem 243: 240-246, 1997.

40. Petiot A, Ogier-Denis E, Blommaart EF, Meijer AJ and Codogno P: Distinct classes of phosphatidylinositol 3'-kinases are involved in signaling pathways that control macroautophagy in HT-29 cells. J Biol Chem 275: 992-998, 2000.

41. Wu YT, Tan HL, Shui G, Bauvy C, Huang Q, Wenk MR, Ong CN, Codogno P and Shen HM: Dual role of 3-methyladenine in modulation of autophagy via different temporal patterns of inhibition on class I and III phosphoinositide 3-kinase. J Biol Chem 285: 10850-10861, 2010.

42. Li Y, Su J, Sun W, Cai L and Deng Z: AMP-activated protein kinase stimulates osteoblast differentiation and mineralization through autophagy induction. Int J Mol Med 41: 2535-2544, 2018.

43. Chen X, Sun K, Zhao S, Geng T, Fan X, Sun S, Zheng M and Jin Q: Irisin promotes osteogenic differentiation of bone marrow mesenchymal stem cells by activating autophagy via the Wnt// $\beta$-catenin signal pathway. Cytokine 136: 155292, 2020.

This work is licensed under a Creative Commons Attribution-NonCommercial-NoDerivatives 4.0 International (CC BY-NC-ND 4.0) License. 